\title{
A calculation of the transport coefficients of hot and dense hadronic matter based on the event generator URASiMA
}

\author{
N. Sasaki ${ }^{\mathrm{a}}$, O. Miyamura ${ }^{\mathrm{b}}$, S. Muroya ${ }^{\mathrm{c} *}$ and C. Nonaka ${ }^{\mathrm{d}}$ \\ a Department of Physics, Hiroshima University, \\ Higashi-Hiroshima 739-8526, Japan \\ ${ }^{\mathrm{b}}$ Department of Physics, Hiroshima University, \\ Higashi-Hiroshima 739-8526, Japan \\ cTokuyama Women's College, Tokuyama, 745-8511, Japan \\ ${ }^{\mathrm{d}}$ Department of Physics, Hiroshima University, \\ Higashi-Hiroshima 739-8526, Japan
}

We evaluate thermodynamical quantities and the transport coefficients of a dense and hot hadronic matter based on the event generator URASiMA (Ultra-Relativistic AA collision Simulator based on Multiple Scattering Algorithm) with periodic boundary conditions. As the simplest example of the transport coefficients we investigate the temperature dependence and the chemical potential dependence of the baryon diffusion constant of a dense and hot hadronic matter.

\section{Introduction}

Physics of a high density and high temperature hadronic matter has been highly studied in the context of both high energy nuclear collisions and cosmology[1]. In recent ultra-relativistic nuclear collisions, though the main purpose should be confirmation of Quark-Gluon Plasma(QGP) state, physics of hot and/or dense hadronic state dominates the system. Hence, thermodynamical properties and transport coefficients of a hadronic matter are essentially important understand the space-time evolution of the exited region. In the cosmology, physics of hot hadrons is important not only for the global evolution of the early universe, but also in the nucleosynthesis problem baryon diffusion would play an important roll.

Because of the highly non-perturbative property of a hot and dense hadronic state, the thermodynamical properties and transport coefficients have not been thoroughly investigated. In this paper, we evaluate the transport coefficients by using statistical ensembles generated by Ultra-Relativistic A-A collision simulator based on Multiple Scattering Algorithm (URASiMA). Originally, the URASiMA is an event generator for the nuclear colli-

*the presenter at this conference 
sion experiments based on the Multi-Chain Model(MCM) of the hadrons [2]. We improved the URASiMA to recover detailed balance at temperature below two hundred $\mathrm{MeV}$, and we can obtain natural thermodynamical behavior without Hagedorn-type strange temperature saturation. This is the first calculation of the transport coefficient of a hot and dense hadronic matter based on an event generator [3].

\section{URASiMA for Statistical Ensembles}

The URASiMA is a relativistic event generator based on hadronic multi-chain model, which aims at describing nuclear-nuclear collision by the superposition of hadronic collisions. Hadronic 2-body interactions are fundamental building blocks of interactions in the model, and all parameters are so designed to reproduce experimental data of hadronhadron collisions. Originally, the URASiMA contains 2-body process (2 incident particle and 2 out-going particles), decay process (1 incident particle and 2 out-going particles), resonance ( 2 incident particles and 1 out-going particle) and production process (2 incident particles and $n(\geq 3)$ out going particles).

The production process is very important for the description of the multiple production at high energies. On the other hand, in the generation of statistical ensembles in equilibrium, detailed balance between processes is essentially important. If re-absorption process does not exist, one-way conversion of energy into particle production occurs rather than heating up and results in artificial saturation of the temperature [4. Therefore, role of re-absorption processes is very important and we should take it into account. However exact inclusion of multi-particle re-absorption processes is very difficult. In order to treat them effectively, multi-particle productions and absorptions are treated as 2-body processes including resonances with succeeding decays and/or preceding formations of the resonances. Here two body decay and formation of resonances are assumed. For example, $N N \rightarrow N N \pi$ is described as $N N \rightarrow N R$ followed by decay of $R \rightarrow N \pi$, where $R$ denotes resonance. The reverse process of it is easily taken into account. In this approach, all the known inelastic cross-sections for baryon-baryon interactions up to $\sqrt{s}<3 \mathrm{GeV}$, are reproduced.

For a higher energy, $\sqrt{s}>3 \mathrm{GeV}$, in order to give appropriate total cross section, we need to take direct production process into account. Only at this point, detailed balance is broken in our simulation; nevertheless, if temperature is much smaller than $3 \mathrm{GeV}$, the influence is negligibly small.

Table 1

Baryons, mesons and their resonances included in the URASiMA.

\begin{tabular}{ccccccccc}
\hline nucleon & $N_{938}$ & $N_{1440}$ & $N_{1520}$ & $N_{1535}$ & $N_{1650}$ & $N_{1675}$ & $N_{1680}$ & $N_{1720}$ \\
$\Delta$ & $\Delta_{1232}$ & $\Delta_{1600}$ & $\Delta_{1620}$ & $\Delta_{1700}$ & $\Delta_{1905}$ & $\Delta_{1910}$ & $\Delta_{1950}$ & \\
meson & $\pi$ & $\eta$ & $\sigma_{800}$ & $\rho_{770}$ & & & & \\
\hline
\end{tabular}


In order to obtain an equilibrium state, we put the system in a box and imposed a periodic condition to the URASiMA as the space-like boundary condition. Initial distributions of particles were given by uniform random distribution of baryons in a phase space. Total energy and baryon number in the box were fixed at initial time and conserved through-out the simulation. Though initial particles were only baryons, many mesons were produced through interactions. After a thermalization time-period of about $100 \mathrm{fm} / \mathrm{c}$, the system seemed to be stationary. In order to confirm the achievement of equilibrium, we calculated energy distributions and particle numbers. Slope parameters of energy distribution of all particles became the same value in the accuracy of statistics. Thus, we may call this value as the temperature of the system. The fact that numbers of species saturate indicates the achievement of chemical equilibrium 3 .

Running the URASiMA many times with the same total energy and total baryons in the box and taking the stationary configuration later than $t=150 \mathrm{fm} / \mathrm{c}$, we obtained statistical ensemble with fixed temperature and fixed baryon number(chemical potential). By using the ensembles obtained through above mentioned manner, we can evaluate thermodynamical quantities and equation of states[5].

\section{Diffusion Constant}

According to the Kubo's Linear Response Theory, the correlation of the currents stands for admittance of the system(first fluctuation dissipation theorem) and equivalently, random-force correlation gives impedance(Second fluctuation dissipation theorem) [6]. As the simplest example, we here focus our discussion on the diffusion constant. First fluctuation dissipation theorem tells us that diffusion constant $D$ is given by current(velocity) correlation,

$D=\frac{1}{3} \int_{0}^{\infty}<\boldsymbol{v}(t) \cdot \boldsymbol{v}\left(t+t^{\prime}\right)>d t^{\prime}$

If the correlation decreases exponentially, i.e., $<\boldsymbol{v}(t) \cdot \boldsymbol{v}\left(t+t^{\prime}\right)>\propto \exp \left(-\frac{t^{\prime}}{\tau}\right)$, with $\tau$ being relaxation time, diffusion constant can be rewritten in a simple form,

$D=\frac{1}{3}<\boldsymbol{v}(t) \cdot \boldsymbol{v}(t)>\tau$.

Because of the relativistic nature of our system, we should use $\boldsymbol{\beta}=\frac{\boldsymbol{v}}{c}=\frac{\boldsymbol{p}}{E}$ instead of $\boldsymbol{v}$ in eq.(11) and $D$ is obtained by,

$$
\begin{aligned}
D & =\frac{1}{3} \int_{0}^{\infty}<\boldsymbol{\beta}(t) \cdot \boldsymbol{\beta}\left(t+t^{\prime}\right)>d t^{\prime} c^{2}, \\
& =\frac{1}{3}<\boldsymbol{\beta}(t) \cdot \boldsymbol{\beta}(t)>c^{2} \tau \\
& =\frac{1}{3}<\left(\frac{\boldsymbol{p}(t)}{E(t)}\right) \cdot\left(\frac{\boldsymbol{p}(t)}{E(t)}\right)>c^{2} \tau
\end{aligned}
$$

with $c$ being the velocity of light. Figure 1 shows correlation function of the velocity of baryons. The figure indicates that exponential damping is very good approximation. Figure 2 displays the our results of baryon diffusion constant in a hot and dense hadronic matter. 

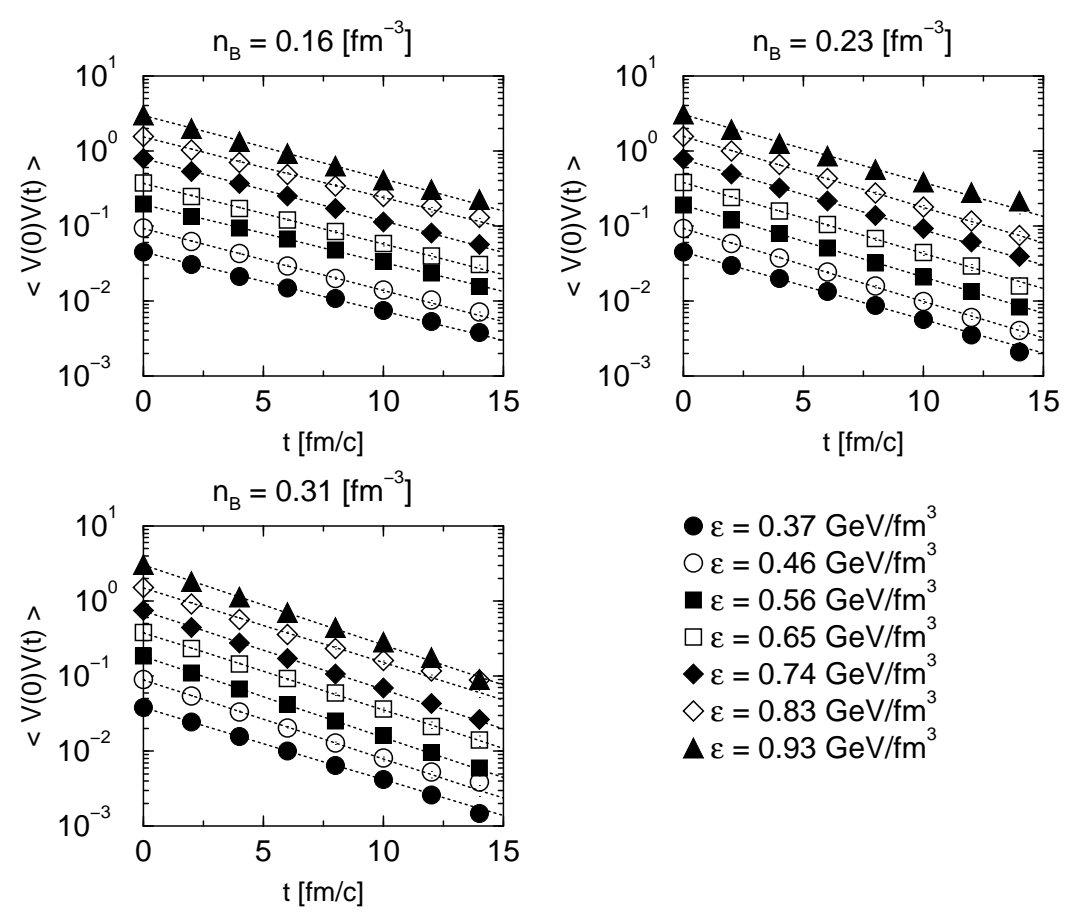

$$
\begin{aligned}
& \bullet \varepsilon=0.37 \mathrm{GeV} / \mathrm{fm}^{3} \\
& \bigcirc \varepsilon=0.46 \mathrm{GeV} / \mathrm{fm}^{3} \\
& \square \varepsilon=0.56 \mathrm{GeV} / \mathrm{fm}^{3} \\
& \square \varepsilon=0.65 \mathrm{GeV} / \mathrm{fm}^{3} \\
& \diamond \varepsilon=0.74 \mathrm{GeV} / \mathrm{fm}^{3} \\
& \diamond \varepsilon=0.83 \mathrm{GeV} / \mathrm{fm}^{3} \\
& \Delta \varepsilon=0.93 \mathrm{GeV} / \mathrm{fm}^{3}
\end{aligned}
$$

Figure 1. Velocity correlation of the baryons as a function of time. Lines correspond to the fitted results by exponential function. Normalizations of the data are arbitrary.

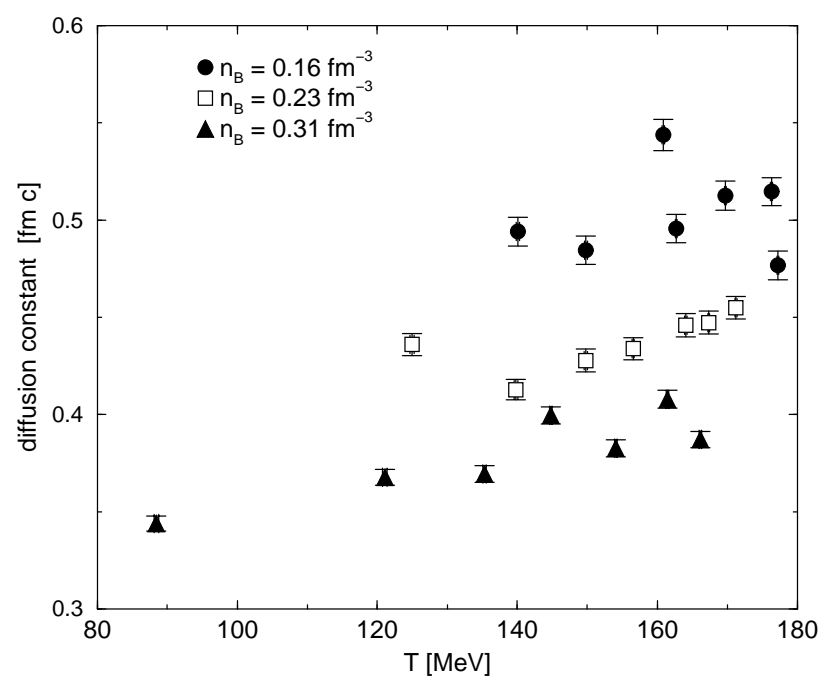

Figure 2. Diffusion constant of baryons. 
Our results show clearer dependence on the baryon number density while dependence on temperature is mild. This result means that, for the random walk of the baryons in our system, baryon-baryon collision process is more important than baryon-meson collisions. In the inhomogeneous big-bang nucleosynthesis scenario, baryon-diffusion plays an important roll. The leading part of the scenario is played by the difference between proton diffusion and neutron diffusion [7]. In our simulation, strong interaction dominates the system and we assume charge independence in the strong interaction, hence, we can not discuss difference between proton and neutron. However obtained diffusion constant of baryon in our simulation can give some kind of restriction to the diffusion constants of both proton and neutron.

\section{Concluding Remarks}

We evaluate diffusion constants of baryons in the hot and dense hadronic matter with use of statistical ensembles obtained by an event generator URASiMA. Our results show clear dependence on baryon number density and weak dependence on temperature. The temperature in our simulation is limited to a small range, i.e., from $100 \mathrm{MeV}$ to $200 \mathrm{MeV}$, and this fact can be one of the reasons for unclear dependence on temperature. Strong baryon number density dependence indicates that, for the baryon diffusion process, baryon plays more important roll than light mesons. In this sense our simulation corresponds to high density region and a non-linear diffusion process occurs.

Calculation of the diffusion constants is the simplest example of first fluctuation dissipation theorem and the entrance of the non-equilibrium physics. From diffusion constant,

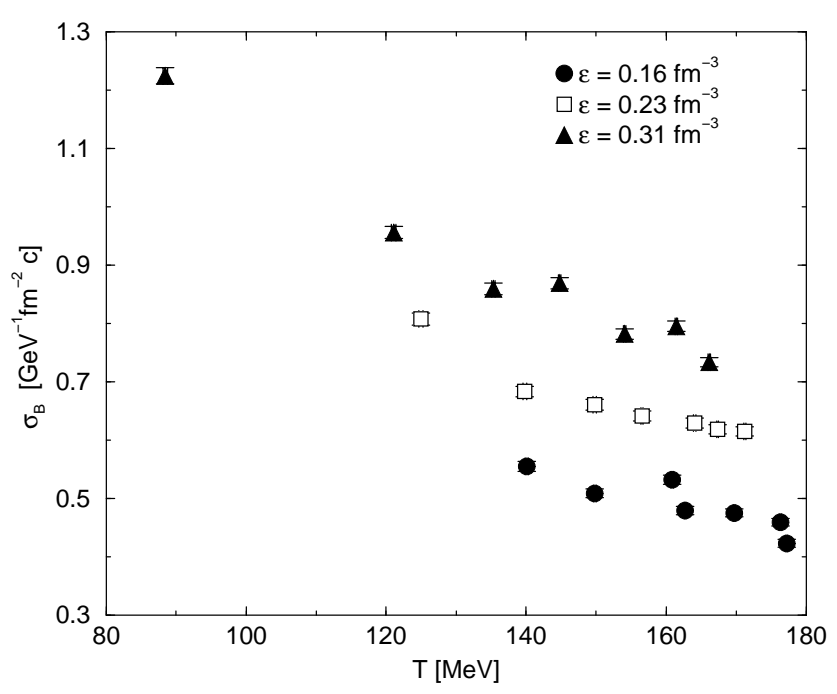

Figure 3. Baryon number charge conductivity as a function of temperature 
we can calculate charge conductivity $[8]$. Figure 3 shows baryon number conductivity $\sigma_{\mathrm{B}}$,

$\sigma_{\mathrm{B}}=\frac{n_{\mathrm{B}}}{k_{\mathrm{B}} T} D$

where $n_{\mathrm{B}}$ is baryon number density, $T$ is temperature and $k_{\mathrm{B}}$ is Boltzmann constant(put as unity through out this paper), respectively. Therefore, if we want, we can discuss Joule heat and entropy production in the Baryonic circuit with use of the above baryon number conductivity.

In principle, taking correlation of appropriate currents, i.e. energy flow, baryon number current, stress-tensor, etc., we can evaluate any kinds of transport coefficients. Though, in relativistic transport theory, there exist several delicate points[9], once we establish phenomenological equations for the high temperature and high density hadronic matter, we can evaluate the appropriate transport coefficients in the same manner. Detailed discussion will be reported in our forthcoming paper.

\section{Acknowledgment}

The authors would like to thank prof. M. Namiki and prof. T. Kunihiro for their fruitful comments. This work is supported by Grant-in-Aid for scientific research number 11440080 of Ministry of Education, Science, Sports and Culture, Government of Japan (Monbusho). Calculation has been done at Institute for Nonlinear Sciences and Applied Mathematics, Hiroshima University.

\section{REFERENCES}

1. For example, see the proceedings of Quark Matter '97, Nucl. Phys. A638(1998)1c.

2. S. Daté, K. Kumagai, O. Miyamura, H. Sumiyoshi and X. Z. Zhang, JPSJ 64(1995)766.

3. N. Sasaki, O. Miyamura, S. Muroya and C. Nonaka, nucl-th/0001013, to appear in Phys. Rev. C; N. Sasaki and O. Miyamura, Prog. Theor. Phys. Suppl. 129(1997)39.

4. S. A. Bass et al., Prog. Part. Nucl. Phys. 41(1998)225; M. Belkacem et al., Phys. Rev. C58(1998)1727.

5. N. Sasaki, in preparation.

6. R. Kubo, Reports on Progress in Physics 29 Part I (1966)255.

7. I. S. Suh and G. J. Mathews, Phys. Rev. D58(1998) 3002.

8. M. Toda, R. Kubo and N. Saito, Statistical Physics I, Springer-Verlag, Berlin, 1992; R. Kubo, M. Toda and N. Hashitsume, Statistical Physics II, Springer-Verlag, Berlin, 1991.

9. M. Namiki and C. Iso, Prog. Theor. Phys. 18(1957)591; C. Iso, K. Mori and M. Namiki, Prog. Theor. Phys. 22(1959)403; L. D. Landau and E. M. Lifshitz, Fluid Mechanics, Pergamon Press., Oxford,1989; S. Weinberg, Astrophys. J. 168(1971)175. 